\title{
Benign Fibrous Histiocytoma of Maxillary Gingiva
}

\section{George A*, Pynadath MK, Jayapalan CS, Noufal A, Manjunath GA and Nair RB}

Department of Oral and Maxillofacial Pathology, MES Dental College, Perinthalmanna, Kerala, India

\begin{abstract}
Background: Fibrohistiocytic lesions are heterogeneous group of mesenchymal tumours that are chiefly composed of fibroblastic and histiocytic elements. They occur commonly in the cutaneous soft tissue of the lower extremities and rarely in the non-cutaneous soft tissues of the head and neck.

Case description: A 37 year old female reported with progressive difficulty in mouth opening. Intraoral examination revealed a large well-defined gingival swelling on the right maxillary tuberosity. The lesion had normal mucosal colouration, granular surface, was non-ulcerated, firm on palpation, and was not associated with tenderness or discharge.

Clinical implication: Benign fibrous histiocytoma $(\mathrm{BFH})$ are uncommon in the oral cavity but should be considered in the differential diagnosis of fibrous growth of gingiva and oral mucosa. Diagnosis of non-cutaneous BFH is difficult because of its non-specific clinical appearance, uncertain histogenesis, microscopic similarities, and lack of any specific immunohistochemical (IHC) marker. To the best of our knowledge this article describes the first case of BFH involving the maxillary posterior buccal gingiva. We also discuss the pathological and IHC characteristics of the lesion.
\end{abstract}

Keywords: Benign fibrous histiocytoma; Fibrohistiocytic; Noncutaneous; Maxillary; Gingiva; Immunohistochemistry; BFH

\section{Introduction}

Benign fibrous histiocytoma (BFH) are fibrohistiocytic tumours composed of biphasic cell population of fibroblasts and histiocytes [1-3]. The true histogenesis of this tumour is uncertain, and most authorities consider these tumours to be of fibroblastic differentiation without any relationship to true histiocytes [1-3]. World Health Organization (WHO) now recognizes the term "fibrohistiocytic" to merely denote a lesion that is composed of cells that resemble round histiocytic and spindled fibroblastic morphology [1-3]. BFH may originate in any part of the body $[1,2]$. They arise commonly in the sun-exposed cutaneous soft tissues of middle aged women $[1,2]$. About $1 \%$ of BFH arise in noncutaneous tissues, mainly in the deep tissues of the lower extremities $[1,2]$. BFH are uncommon in the head and neck region [1,2]. Skoulakis et al. states that on excluding fibrohistiocytic lesions of the facial bones, less than 10 cases of non-cutaneous BFH of the head and neck have been reported in the English literature [4]. BFH are rare in the oral cavity, with few reported cases in the buccal mucosa, vestibule, tongue, soft palate, jaw bones, floor of the mouth, and gingiva [1]. Arriving at a diagnosis of BFH is difficult as they appear clinically as non-specific, painless, solitary, slowly enlarging swelling that may affect the normal physiology and function of the area in which they arise [1,5]. The lesion is frequently reported after a scalpel-biopsy through a combination of light microscopic and immunohistochemical (IHC) examination. BFH has no known specific IHC marker and the diagnosis is usually based on absence of markers [1,4]. A search of English language medical literature returned a total of two case reports of $\mathrm{BFH}$ in the gingiva, both involving the lingual mandibular gingiva $[3,5]$. To the best of our knowledge this article describes the first case-report of $\mathrm{BFH}$ involving the maxillary posterior buccal gingiva. We also attempt to discuss the pathological and IHC characteristics of the lesion.

\section{Case Description}

A 37 year old housewife presented to our teaching and research institute with a three month clinical history of progressive difficulty in mouth opening. She was otherwise healthy and gave no relevant medical history of any systemic disease or medication. No clinical history of trauma to the jaw, surgical intervention, or any recent dental procedures were given. A physical and extraoral examination revealed no relevant abnormal findings. Tempero-mandibular joint (TMJ) examination elicited no tenderness, clicking, or any other relevant findings. Her interincisal mouth opening was measured as $29 \mathrm{~mm}$. Intraoral examination revealed a poor oral hygiene index and a high caries index with multiple decayed teeth including root-stumps of upper and lower posterior teeth. Soft tissue examinations revealed a large well-defined gingival swelling of $2 \times 3 \mathrm{~cm}$ on the buccal aspect of right maxillary tuberosity extending to the first molar. The gingival mass had normal mucosal colouration, had granular surface, was non-ulcerated, firm to palpation, and was not associated with tenderness or discharge. The adjacent second molar was grossly decayed with pulpal exposure and grade II mobility with pain on horizontal and vertical percussion. Cervical lymph nodes were not palpable. Investigatory orthopentograph revealed multiple decayed teeth with pulpal exposure and few with periapical pathology. No TMJ or associated bony pathology was identified. A clinical diagnosis of gingival fibroma obstructing coronoid movement was given. She was advised and underwent surgical excision of the gingival mass under local anaesthesia along with removal of right maxillary third molar root-stump and the second molar. Post surgically her chief complaint was resolved. The haematoxylin and eosin (H\&E) stained histopathological sections showed a non-infiltrating fibrohistiocytic lesion with grenz zone in relation to an overlying stratified squamous surface epithelium. The lesion was chiefly composed of interlacing fascicles of spindle cells having plump and vesicular nucleus with tapered and blunt ends arranged in a storiform pattern (Figure 1). Focal areas of few pleomorphic cells, round histiocytes, lipid laden macrophages, lymphocytes, and multinucleated giant cells were noted (Figure 2). No necrosis was present and the mitotic count was less

*Corresponding author: Antony George, Department of Oral and Maxillofacial Pathology, MES Dental College, Perinthalmanna, Malappuram District, 679338. Kerala India, Tel: +919895889453; Email: drantgeo@gmail.com

Received February 26, 2014; Accepted March 26, 2014; Published March 28, 2014

Citation: George A, Pynadath MK, Jayapalan CS, Noufal A, Manjunath GA, et al. (2014) Benign Fibrous Histiocytoma of Maxillary Gingiva. Dentistry 4: 227. doi:10.4172/2161-1122.1000227

Copyright: $\odot 2014$ George A, et al. This is an open-access article distributed under the terms of the Creative Commons Attribution License, which permits unrestricted use, distribution, and reproduction in any medium, provided the original author and source are credited. 


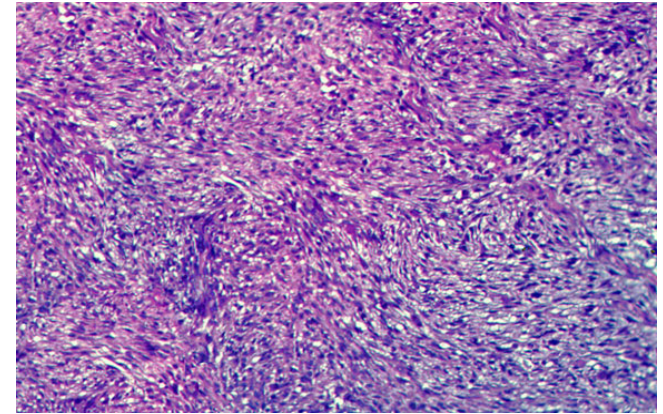

Figure 1: Histopathological section (10x magnification) - fibrohistiocytic lesion with interlacing fascicles of spindle cells arranged in storiform pattern.

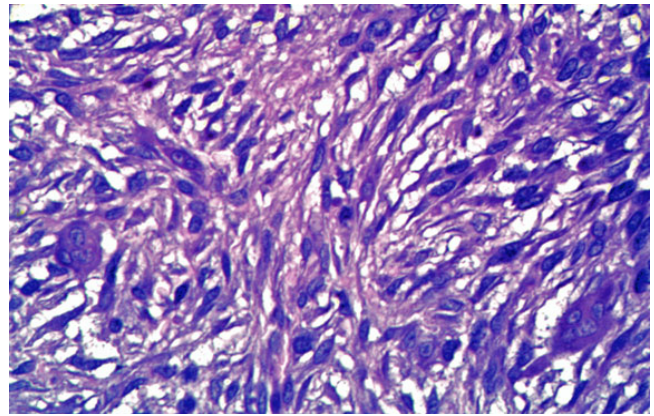

Figure 2: Histopathological section (40x magnification) - fibrohistiocytic lesion with few pleomorphic spindle cells, xanthoma cells, and multinucleated giant cells.

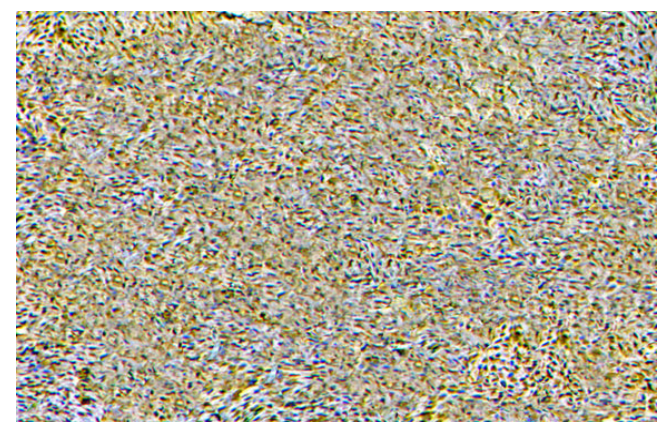

Figure 3: Immunohistochemistry - positive immunoreactivity to vimentin.

than one per 10 high-power fields. A provisional diagnosis of $\mathrm{BFH}$, pleomorphic undifferentiated sarcoma, fibrosarcoma, neurofibroma, solitary fibrous tumour (SFT), and leiomyoma was made and the tissue-blocks were forwarded for immunohistochemical (IHC) studies to arrive at a confirmatory diagnosis. The tumour cells showed strong immunoreactivity for vimentin (Figure 3) and CD 68 (Figure 4), focal immunoreactivity for smooth muscle actin (SMA) (Figure 5), and negative immunoreactivity for desmin, S-100, and Ki-67. Based on the $\mathrm{H} \& \mathrm{E}$ and IHC findings a final diagnosis of BFH was made. After 18 months of periodic follow-up, the patient is asymptomatic with no evidence of tumour recurrence. A written informed patient consent was obtained to publish her clinical details and investigatory findings for academic advancement without revealing her personal identity.

\section{Discussion}

BFH was not known as a clinical entity before 1960 because of confusion over the natural history of this fibrohistiocytic lesion $[1,4]$.
Electron microscopic studies have shown two distinct cell types in these tumours. One end of the spectrum has cells resembling fibroblasts with organized lamellae of rough endoplasmic reticulum, few or no lipid droplets, and no phagolysosomes [2]. The other end of the spectrum has rounded cells resembling histiocytes with numerous cell processes, mitochondria, and phagolysosomes [2]. BFH is academically separated into (1) cutaneous type (synonym: dermatofibroma), that arise in the sun-exposed areas of the skin, and the rare (2) non-cutaneous type which arises in the deep soft tissues of the extremities [1,4]. The rare oral $\mathrm{BFH}$ tend to occur in middle-aged and older adults with a mean age of $40 \mathrm{yr}$ and a male-female ratio of $2.5: 1[5,6]$. They clinically appear as painless slow growing mass that cause progressive changes at the involved site $[5,6]$. They vary in size from few $\mathrm{mm}$ to several $\mathrm{cm}$ and have shown a pre-operative duration of 3-12 months [5,6]. The tumour is usually associated with a history of antecedent trauma or chronic infection, suggesting a reactive disease $[1,4]$.

Histopathologically BFH show a dual population of histiocytic and fibrous elements, with most researchers now believing that the histiocyte-like cells originate from undifferentiated mesenchymal stem-cells and are of fibroblastic origin [1-4]. BFH is characterised by cellular proliferation of spindle shaped fibroblasts with vesicular nucleus arranged in short intersecting fascicles of storiform pattern [1-6]. Round histiocyte-like cells, lipid-containing xanthoma cells, multinucleated giant cells, and scattered lymphocytes are frequently seen [1-6]. Our case showed similar microscopic features, with positive immunoreactivity for vimentin (Figure 3) and CD 68 (Figure 4) demonstrating that the lesion is composed of fibroblastic and histiocyte-like cells. The negativity for S-100 differentiated the lesion from neurogenic tumours, and the negativity for Ki-67 demonstrated that the lesion was not aggressive or proliferative. It is important to differentiate $\mathrm{BFH}$ from aggressive neoplasms including the more frequent undifferentiated pleomorphic sarcoma (synonym: malignant

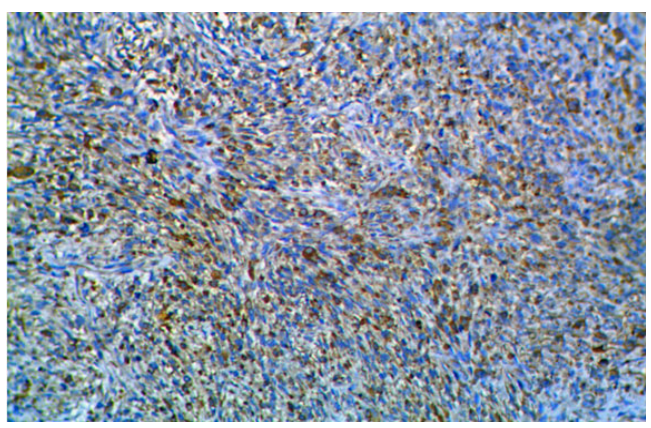

Figure 4: Immunohistochemistry - positive immunoreactivity to CD 68.

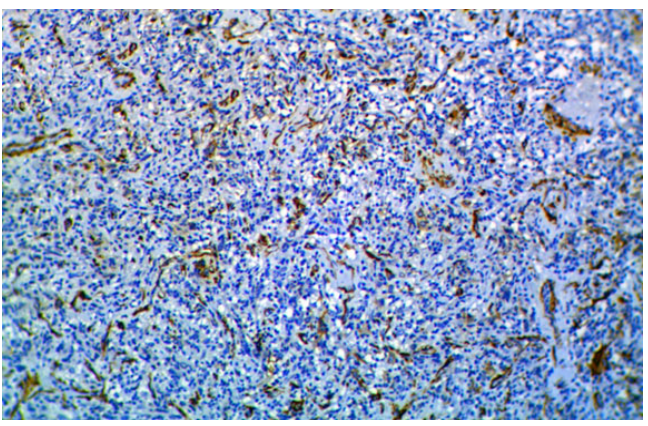

Figure 5: Immunohistochemistry - focal immunoreactivity of blood vessels to smooth muscle actin (SMA). 
fibrous histiocytoma) and fibrosarcoma [5]. Undifferentiated pleomorphic sarcoma was excluded due to lack of pleomorphic sarcomatous cells, lack of typical and atypical mitotic figures, and lack of bizarre giant cells, necrosis, and prominent areas of haemorrhage [7]. Fibrosarcoma was excluded by the lack of obvious malignant features, invasive margins, and the characteristic herring-bone pattern [6]. SFT was excluded as they pathologically appear as monomorphic spindle cells organised into interlacing fascicles and 'pattern-less' pattern with hemangiopericytoma-like vascular spaces [8]. They show positive immunoreactivity to vimentin and negative immunoreactivity to desmin and SMA [8]. Our case showed SMA immunoreactivity for the blood vessels associated with the tumour, the true lesional cells showed negative immunoreactivity (Figure 5). We excluded AFH as it occurs in children and young adults, is characterized predominantly by sheets of histiocytic cells arranged in serpentine pattern having cystic areas of haemorrhage $[2,9,10]$. The lesion is characteristically surrounded by dense lymphocytic stroma and plasma cells, and lack giant cells and xanthoma cells $[2,9,10]$. According to Weiss et al. SMA may occasionally be positive in $\mathrm{BFH}$ and their sporadic immunoreactivity should not considered as evidence of smooth muscle neoplasm [2]. Leiomyoma was excluded as they have a more distinct fascicular arrangement with blunt-ended plumper nucleus and cytoplasm showing longitudinal striations corresponding to myofilamentous material [2]. They strongly express SMA unlike the focal immunoreactivity noted in BFH [2].

The treatment of choice for oral BFH is en-block surgical excision. The prognosis is good and is without recurrence when completely excised [4,5]. However, it is recommended to have regular periodic follow-up because of the difficulty in arriving at the diagnosis and of the few reported cases of recurrence, possibly due to inadequate margins of resection [4,5]. Our case underwent complete surgical excision and post 18 months follow-up the patient is asymptomatic.

\section{Conclusion}

We have reported the clinical, microscopic, and immunohistochemical aspects of non-cutaneous BFH afflicting the maxillary posterior buccal gingiva. Oral BFH mimics many clinical oral lesions and may often be misdiagnosed initially. It may represent a pathologist's nightmare since the histopathological and morphological patterns are common to many other soft tissue tumours. As there are no known specific IHC marker BFH essentially represents a diagnosis of exclusion. Oral BFH are rare but it should be considered in the differential diagnosis of fibrous growth of gingiva and oral mucosa.

\section{Authors Contributions}

AG performed the pathological examination of the lesion and along with MKP was the major contributor in writing the manuscript. CSJ and AN contributed by reviewing the literature and helped in drafting the manuscript. GAM and RBN performed the surgical procedure and contributed to the expatiation of the clinical data. All the authors read and approved the final manuscript.

\section{References}

1. Giovani P, Patrikidou A, Ntomouchtsis A, Meditskou S, Thuau H, et al. (2010) Benign Fibrous Histiocytoma of the Buccal Mucosa: Case Report and Literature Review. Case Rep Med 2010: 306148.

2. Weiss WS, Goldblum JR (2008) Benign Fibrohistiocytic Tumors: Enzinger and Weiss's Soft Tissue Tumors. (5thedn), Mosby Elsevier, China.

3. Rajathi P, Jacob M, Priyadharshini I, Sekar B (2013) Benign fibroushistiocytoma of the gingiva. J Pharm Bioallied Sci Suppl 2: S166-168.

4. Skoulakis CE, Papadakis CE, Datseris GE, Drivas El, Kyrmizakis DE, et al. (2007) Subcutaneous benign fibrous histiocytoma of the cheek. Case report and review of the literature. Acta Otorhinolaryngol Ital 27: 90-93.

5. Menditti D, Laino L, Mezzogiorno A, Sava S, Bianchi A, et al. (2009) Oral benign fibrous histiocytoma: two case reports. Cases J 2: 9343.

6. Neville BW, Damm DD, Allen CM, Bouquot JE (2009) Soft Tissue Tumors: Oral and Maxillofacial Pathology. (3rdedn), Saunders, Philadelphia.

7. Matushansky I, Charytonowicz E, Mills J, Siddiqi S, Hricik T, et al. (2009) MFH classification: differentiating undifferentiated pleomorphic sarcoma in the 21st century. Expert Rev Anticancer Ther 9: 1135-1144.

8. Manor E, Sion-Vardy N, Woldenberg Y, Bodner L (2012) Solitary Fibrous Tumor of the Buccal Vestibule: Report of Two Cases. J Maxillofac Oral Surg 11: 323327.

9. Xiang L, Zhou J, Gu W, Yang B (2013) Retroperitoneal angiomatoid fibrous histiocytoma: A case report and review of the literature. Oncol Lett 5: 18331835.

10. Chen G, Folpe AL, Colby TV, Sittampalam K, Patey M, et al. (2011) Angiomatoid fibrous histiocytoma: unusual sites and unusual morphology. Modern Pathol 24 1560-1570. 